\title{
Uso de evaluación electrónica en exámenes clínico objetivo estructurado
}

\author{
Use of electronic evaluation in objective \\ structured clinical examinations
}

Carlos Orellano,* Jorge Huerta-Mercado ${ }^{\ddagger}$

\section{Palabras clave: Examen clínico objetivo estructurado, evaluación electrónica. \\ Keywords: Objective structured clinical examination, electronic assessment.}

\section{RESUMEN}

Introducción: Una de las dificultades del examen clínico objetivo estructurado (ECOE) es la gestión de datos de evaluación. Objetivo: Describir el uso de la evaluación electrónica en nuestro dos primeros ECOEs con la tecnología disponible en la Facultad de Medicina de la Universidad Peruana Cayetano Heredia. Material y métodos: Se utilizó la plataforma Moodle para la creación de rúbricas electrónicas. Los evaluadores recibieron capacitación en su uso. En el primer ECOE se colocaron laptops en cada estación para hacer la evaluación electrónica por Wi-Fi. Para el segundo ECOE se corrigieron las dificultades tecnológicas ocurridas en el primero y se identificarían la ocurrencia de nuevos problemas. Resultados: Se logró gestionar los datos de evaluación de acuerdo con la tríada de seguridad de información y no ocurrieron problemas técnicos que obligaran a detener el primer ECOE. Se completaron todas las rúbricas electrónicas y los evaluadores coincidieron en que fue sencillo trabajar con ellas. Sin embargo, hubo dificultades con las calificaciones reportadas y posiblemente el uso de laptops podría interferir con la concentración de los estudiantes. Para el segundo ECOE se corrigió la forma de llenado de las rúbricas y se utilizaron tabletas. Al no presentarse nuevos problemas con la gestión de información se consideró este método de trabajo aceptable para los futuros ECOEs. Conclusiones: Fue posible adaptar los recursos tecnológicos disponibles para gestionar las evaluaciones en los ECOEs.

\section{ABSTRACT}

Introduction: One of the difficulties of the objective structured clinical examination (OSCE) is the management of assessment data. Objective: Describe the use of electronic evaluation in our first two OSCEs with the available technology in the School of Medicine at Cayetano Heredia Peruvian University. Material and methods: Moodle platform was utilized to create electronic rubrics. The evaluators received training in its use. In the first OSCE, laptops were placed at each station to do electronic evaluation via Wi-Fi. For the second OSCE, the technological difficulties occurred in the first were corrected and the occurrence of new problems would be identified. Results: Evaluation data was managed according to the information security triad and there were no technical problems that forced the first OSCE to stop. All electronic rubrics were completed and evaluators coincided with easy use of them. However, there were difficulties with the reported grades and possibly the use of laptops could interfere with student concentration. For the second OSCE, the way of filling the rubrics was corrected and tablets were used. In the absence of new problems with information management, this method of work was considered acceptable for future OSCE. Conclusions: It was possible to adapt the available technological resources to manage the evaluations in the OSCE.

\section{INTRODUCCIÓN}

* Docente del Departamento de Clínicas Médicas. ₹ Jefe del Área Clínica y Prácticas Preprofesionales.

Facultad de Medicina. Universidad Peruana Cayetano Heredia.

Recibido: 11/11/2019 Aceptado: 13/07/2020

doi: $10.35366 / 95227$
L a simulación se ha ido incorporando en el currículo médico como parte del entrenamiento para el desarrollo de competencias requeridas en el contexto profesional y posibilita el diseño de evaluaciones más controladas y objetivas; en contraste con los exámenes clínicos tradicionales (ECTs), en los cuales la seguridad del paciente podría vulnerarse. Por ello, en la Facultad de Medicina de la Universidad Peruana Cayetano Heredia se instauró el Examen Clínico Objetivo Estructurado (ECOE) para evaluar el desempeño.
Realizar un ECOE por primera vez es una tarea compleja que consume mucho tiempo y requiere del desarrollo de estructuras académicas y administrativas. ${ }^{1}$ Durante la planificación del primer ECOE de nuestra facultad en el año 2014, surgieron varias interrogantes y una de ellas fue cómo gestionar los datos de evaluación de los estudiantes. En los anteriores ECTs se utilizaba una única rúbrica en papel por alumno y posteriormente se procedía a la consolidación de datos en una hoja de cálculo para obtener las calificaciones. En cambio, cada estación del ECOE posee su propio formulario de evaluación. 
Continuar con la forma de gestión de datos de los ECTs en el ECOE resultaría en: (a) usar más de un millar de unidades de papel, (b) rúbricas incompletas o pérdida de éstas, (c) mayor tiempo para elaborar las hojas de cálculo y (d) aumento del riesgo de errores de tipeo. Una opción era recopilar los datos cuanto antes para detectar y corregir cualquier error mientras se desarrolla el ECOE. ${ }^{2}$ Sin embargo, no se contaba con más personal para hacerse cargo de dicha tarea, por lo que fue necesario buscar otra solución.

Las tecnologías de información y comunicaciones facilitan el manejo de gran cantidad de datos, y la evaluación electrónica es una de las áreas de aplicación en educación. ${ }^{3}$ Hasta antes de nuestra implementación, había pocos estudios sobre evaluación electrónica en ECOEs. Schmidts menciona que con el uso de PDAs (Personal Digital Assistants) aumentó la velocidad de captura de datos, facilitó la evaluación de los datos y mejoró la retroalimentación. ${ }^{4}$ Más adelante, Treadwell comparó el uso de PDAs con el método en papel y sus hallazgos señalaron que la forma electrónica era tan efectiva y más eficiente (en términos de menor consumo de tiempo), ${ }^{5}$ siendo altamente aceptada por los evaluadores, quienes la encontraron menos invasiva y les permitió tener más tiempo para observar el desempeño de los estudiantes.

En nuestra situación, incorporar la evaluación electrónica estaba entre decidir crear un aplicativo, adquirir alguna solución informática en ECOEs o adaptar uno de los programas utilizados en la facultad (software a medida, estándar o híbrido, respectivamente). La primera alternativa fue descartada, ya que el tiempo requerido sobrepasaba al plazo disponible. La segunda tampoco era viable porque acarrearía una inversión económica no contemplada en el presupuesto.

Tabla 1: Pasos para configurar Moodle para el examen clínico objetivo estructurado.

1. Crear un nuevo curso y, en sus ajustes, seleccionar "grupos separados" en la opción "modo de grupo" para habilitar el filtro de miembros

2. Agregar una "tarea" para cada estación y en configuración escoger "rúbricas" como método de calificación

3. Elaborar los formularios de calificación con el editor de rúbricas

4. Crear las cuentas de usuario para los evaluadores y añadirlos dentro del curso como "docente no editor" por defecto

5. Asignar a los evaluadores a sus respectivas rúbricas como "docente” (rol con permisos para calificar) a través de la opción "roles asignados localmente"
Los programas informáticos para ECOEs incluyen un generador de rúbricas, módulos de administración de usuarios y de grupos, y un libro de calificaciones; funciones también disponibles en plataformas web de gestión educativa convencionales como Moodle, un software abierto, el cual es utilizado en nuestra facultad desde hace muchos años. ${ }^{6}$ Tras revisar la documentación de uso del software, optamos por acondicionarlo para gestionar las evaluaciones del ECOE.

El objetivo de este artículo es describir nuestra experiencia sobre el uso de la evaluación electrónica en nuestro primer ECOE a partir de las herramientas informáticas disponibles y las correcciones realizadas para el segundo ECOE.

\section{MATERIAL Y MÉTODOS}

Los dos primeros ECOEs se llevaron a cabo en el Centro de Simulación de la Facultad de Medicina de la Universidad Peruana Cayetano Heredia en el año 2014. Estaban dirigidos a 130 estudiantes del cuarto año del pregrado de medicina que llevaban el curso de Introducción a la Clínica.

El primer ECOE se realizó en la última semana del mes de mayo. Estaba conformado por 11 estaciones, siendo la evaluación en nueve de éstas a través de rúbricas. Hubo una rúbrica diseñada específicamente para cada estación cuya estructura difería de las demás. La rúbrica consistía en una lista de cotejo no binaria (ítems con diferentes escalas multidimensionales) para el puntaje de la estación (PE) y una escala de valoración global (EVG). ${ }^{7}$ Con el PE y la EVG se aplicaría el método de regresión límite (MRL). ${ }^{8}$ Los alumnos fueron distribuidos a conveniencia en 15 grupos (13 grupos de nueve alumnos, un grupo de ocho alumnos y un grupo de cinco alumnos). A cada grupo se le asignó una ronda de estaciones. Por cada ronda se hizo una selección previa, también a conveniencia, de nueve estaciones y heterogenia entre sí.

En Moodle se creó un curso virtual y se hicieron las configuraciones respectivas (Tabla 1). Para que los evaluadores pudieran calificar a los estudiantes se hizo una "inscripción masiva" de estos al aula virtual subiendo un archivo CSV que contenía los códigos de identificación (única forma de reconocimiento, ya que no se utilizaron nombres ni apellidos) y grupos.

Dos días antes del primer ECOE, los evaluadores fueron convocados para recibir una capacitación presencial de una hora sobre el llenado de las rúbricas electrónicas en una sala 


\begin{tabular}{lll}
\hline \multicolumn{2}{c}{ Tabla 2: Seguridad de la información en Moodle. } \\
\hline & Definición & Moodle \\
\hline Confidencialidad & $\begin{array}{l}\text { El acceso a la información es para usuarios } \\
\text { autorizados } \\
\text { Integridad }\end{array}$ & Sólo usuarios autorizados modifican los datos evaluadores pueden acceder al curso \\
Disponibilidad & $\begin{array}{l}\text { La información se entrega cuando } \\
\text { es requerida }\end{array}$ & $\begin{array}{l}\text { Una rúbrica sólo se completa o modifica por } \\
\text { su respectivo evaluador } \\
\text { Una rúbrica electrónica completada puede } \\
\text { revisarse inmediatamente después de que el } \\
\text { evaluador la haya enviado }\end{array}$ \\
\hline
\end{tabular}

de reuniones y se les hizo una demostración por proyector del manejo de la plataforma. A diferencia de los reportes previos y por el avance tecnológico, dentro de cada estación se colocó una laptop sobre una mesa para que el evaluador accediera a la plataforma por conexión Wi-Fi. Por precaución, se dejaron rúbricas impresas en las estaciones. Al concluir el ECOE se descargarían los resultados de cada estación en hojas de cálculo para realizar el MRL y otros análisis, y además se identificarían los problemas relacionados en la gestión de información.

El segundo ECOE se programó luego de un mes. Ninguna estación del primero se repitió en el segundo; por tanto, se crearon nuevas rúbricas en una nueva aula virtual. Para este ECOE se diseñaron 13 estaciones y, a diferencia del anterior, estuvieron incluidos en todas las rondas. Del total de estaciones, 11 se evaluaron con rúbricas. Los alumnos fueron distribuidos en 10 grupos de 13 miembros a conveniencia. En la planificación del ECOE se corregirían las dificultades tecnológicas resultantes del primero. Al finalizar, se enumerarían los nuevos problemas.

\section{RESULTADOS}

A la capacitación asistieron seis de los nueve evaluadores, ésta se extendió media hora más por algunas dudas específicas de cada estación. Los otros tres evaluadores recibieron una breve inducción de aproximadamente 10 minutos antes de iniciar la primera ronda del ECOE.

Con las rúbricas en Moodle se logró gestionar los datos de evaluación de acuerdo con la tríada de seguridad de información (Tabla 2). No ocurrieron problemas técnicos que hayan obligado a interrumpir el primer ECOE. Todas las rúbricas electrónicas fueron completadas, ya que Moodle alertaba al evaluador si había quedado algún ítem vacío al resaltarlo. En total, se llenaron 919 rúbricas electrónicas y todos los alumnos participaron en el ECOE.

Sin embargo, hay que señalar que hubo dificultades con el manejo de los PEs informados en el libro de calificaciones. Como las EVGs se incluyeron dentro de los formularios de las rúbricas, Moodle las consideró como parte de los elementos para calcular los PEs y no había manera de excluirlas desde el front-end para volver a calcular las calificaciones, lo cual impidió aplicar el MRL. En consecuencia, se requirió tiempo adicional en la revisión de la base de datos de Moodle para extraer los puntajes en hojas de cálculo y obtener los PEs corregidos.

Por otro lado, los evaluadores opinaron que les resultó sencillo trabajar con las rúbricas electrónicas, pero acotaron que parecería importante el tipo de dispositivos usados para la evaluación electrónica. Notaron que la presencia de laptops dentro de las estaciones distraía a algunos estudiantes y que tal vez podrían interferir en su rendimiento. Además, al estar colocadas sobre mesas, ocupaban espacio y serían de alguna manera incompatibles con el entorno simulado.

Pese al problema de las rúbricas y los comentarios de los evaluadores, se continuó usando Moodle en el segundo ECOE haciendo algunas modificaciones. Ya no se creó una única "tarea" por cada estación, sino que se hicieron dos: una para el listado de cotejo y otra para la EVG. También, se reemplazaron las laptops por tabletas para ofrecer una mejor maniobrabilidad al tener bajo peso y se retiraron las mesas de las estaciones.

Durante el desarrollo de dicho ECOE tampoco ocurrieron incidentes técnicos que llevaran a detenerlo. El libro de calificaciones mostraba los PEs seguidos de su respectiva EVG, por lo que sólo requerimos hacer la descarga de la hoja de cálculo. Los evaluadores manifestaron que las menores dimensiones de las tabletas hicieron que su presencia sea menos invasiva, sin interrumpir 
la concentración de los estudiantes. Todos los estudiantes fueron evaluados y se obtuvieron 1,430 rúbricas completas.

Al no presentarse nuevos problemas con la gestión de información, se consideró este método de trabajo con software híbrido aceptable para los futuros ECOEs.

\section{DISCUSIÓN}

Optar por la evaluación electrónica en el primer ECOE y continuar con ella en el segundo fue una decisión acertada. Se evitó el tiempo invertido, gastos en material y los potenciales errores de recolección propios de la metodología en papel. A pesar de que han transcurrido varios años desde que hicimos esta implementación, no hay en la literatura artículos que describan el uso de software híbrido (menos aún Moodle) para la evaluación en ECOEs, por lo que consideramos importante reportar nuestra experiencia.

Podemos indicar que, tras una revisión posterior, encontramos nuevos reportes. $\mathrm{Al}$ año siguiente de iniciar nuestros ECOEs, se publicó un estudio sobre uso de laptops por Meskell y colegas, quienes también afirman que se les facilitó el almacenamiento y análisis de datos, con entrega de retroalimentación mucho más a tiempo y una alta satisfacción de parte de los evaluadores. ${ }^{9}$ Luimes y Labrecque, quienes condujeron la evaluación electrónica con tabletas, destacan que la evaluación electrónica mejora la eficiencia, objetividad y precisión de los ECOEs. ${ }^{10}$ Recientemente, Daniels y colaboradores lograron una reducción de formularios incompletos o sin comentar con iPads y mayor cantidad de palabras en los comentarios a comparación del método en papel en un ECOE dirigido a residentes de medicina interna. ${ }^{11}$ Además, al reducirse el tiempo de entrega de resultados y permitir a los estudiantes escribir un plan de aprendizaje en un siguiente ECOE promovió en ellos cambios conductuales.

Acerca de las desventajas con tabletas, Snodgrass y su equipo indicaron que los evaluadores participantes de su estudio mencionaron dificultades relativas al uso de iPads. ${ }^{12}$ En nuestro caso, los evaluadores fueron médicos egresados de nuestra facultad de los últimos cinco años como máximo, pertenecientes a la generación de los "nativos tecnológicos", cuyo aprendizaje en el uso de dispositivos sería mucho más rápido. Por otro lado, Judd y su grupo describen el proceso de asimilación tecnológica de iPads en
ECOEs y minientrevistas múltiples; sin embargo, descartaron usar Wi-Fi por la ocurrencia de fallas técnicas (pérdidas intermitentes de conexión) en su primera fase. ${ }^{13} \mathrm{~A}$ diferencia de ellos, nosotros nos aseguramos que la cobertura de la señal inalámbrica alcanzara a todas las estaciones en el Centro de Simulación antes de ejecutar los ECOEs. Además, contábamos con una red no saturada dentro del campus universitario.

En cuanto a limitaciones, no se pudo comparar entre el llenado de rúbricas en papel y las rúbricas electrónicas en los ECOEs, debido a que en nuestra facultad no se han realizado este tipo de evaluaciones previamente. Monteiro y colaboradores hallaron que la transición desde tarjetas ópticas a tabletas podría afectar la varianza de los puntajes de las estaciones, ${ }^{14}$ pero sin estar influenciada por el sesgo sistemático y al no encontrarse cambios significativos en la fiabilidad general no se estaría comprometiendo los estándares de evaluación. Al contrario, Schmitz y su grupo habían reportado previamente que las calificaciones no estarían influenciadas por el uso de iPads. ${ }^{15}$

Otra limitación fue que no se midió el grado de aceptabilidad de parte de los evaluadores de forma objetiva por un estudio cuantitativo mediante cuestionarios como el Technology Acceptance Mode/ ${ }^{16,17}$ o cualitativo por grupos focales o entrevistas en profundidad. Para este fin, se podría seguir la metodología hecha por Currie y colegas, quienes hallaron una alta aceptabilidad en evaluadores; ${ }^{18}$ o la realizada por Hochlehnert y su equipo, quienes además recogieron información de los estudiantes y encontraron también una alta aceptación en ambos grupos. ${ }^{19}$

Lo que nos quedó pendiente, por no haber sido contemplada en primera instancia en nuestra implementación, fue brindar retroalimentación, ya que esta última es vital para fomentar un mejor desempeño en los estudiantes, $y$ hacerlo de forma electrónica. Al respecto, Denison y colaboradores encontraron que en ECOEs formativos los evaluadores ingresaron mayor número de comentarios (como retroalimentación) y hubo una mejor calidad de estos a partir de que utilizaron iPads en comparación a los escritos en papel. ${ }^{20}$ Pese a que Munro y sus colegas también encontraron mayor cantidad de retroalimentación por iPads, no existió diferencias en su calidad. ${ }^{21}$ Harrinson y su grupo de investigación destacan las grabaciones de audio por iPads como medio para brindar retroalimentación electrónica. ${ }^{22}$ Ashby y su equipo sugieren que no sólo debe capacitarse a los eva- 
luadores en el uso del software, sino también en dar retroalimentación de forma efectiva a través de la tecnología, ya que es altamente valorada por los estudiantes. ${ }^{23}$ Aunque estos reportes indican que se utilizó software a medida, se podría averiguar en una próxima implementación la posibilidad de hacerlo con aplicaciones híbridas.

\section{CONCLUSIONES}

Es posible adaptar los recursos tecnológicos disponibles para la evaluación electrónica, específicamente en los ECOEs. Se evitó la laboriosa tarea de recopilar datos con rúbricas en papel. Las rúbricas electrónicas nos permitieron hacer un mejor uso del tiempo, enfocándolo principalmente en el análisis de datos. Tal es así que seguimos utilizándolas. Compartimos nuestra experiencia con la intención de que otras escuelas de medicina con recursos limitados para desarrollar o comprar software especializado para este tipo de evaluaciones puedan replicarla.

\section{REFERENCIAS}

1. Khan KZ, Ramachandran S, Gaunt K, Pushkar P. The Objective Structured Clinical Examination (OSCE): AMEE Guide No. 81. Part I: an historical and theoretical perspective. Med Teach. 2013; 35 (9): e1437-1446.

2. Zabar S, Kachur EK, Kalet A, Hanley K. Objective structured clinical examinations: 10 steps to planning and implementing OSCEs and other standardized patient exercises. New York: Springer; 2013, p. 92.

3. Ellaway R, Masters K. AMEE Guide 32: e-Learning in medical education Part 1: Learning, teaching and assessment. Med Teach. 2008; 30 (5): 455-473.

4. Schmidts MB. OSCE logistics--handheld computers replace checklists and provide automated feedback. Objective structured clinical examination. Med Educ. 2000; 34 (11): 957-958.

5. Treadwell I. The usability of personal digital assistants (PDAs) for assessment of practical performance. Med Educ. 2006; 40 (9): 855-861.

6. Orellano C. Uso de los espacios virtuales para la docencia en cursos de pregrado de medicina. Rev Medica Hered. 2012; 23 (3): 188.

7. Khan KZ, Gaunt K, Ramachandran S, Pushkar P. The Objective Structured Clinical Examination (OSCE): AMEE Guide No. 81. Part II: organisation \& administration. Med Teach. 2013; 35 (9): e1447-1463.

8. Kramer A, Muijtjens A, Jansen K, Düsman H, Tan L, Van der Vleuten C. Comparison of a rational and an empirical standard setting procedure for an OSCE. Objective structured clinical examinations. Med Educ. 2003; 37 (2): 132-139.

9. Meskell P, Burke E, Kropmans TJB, Byrne E, Setyonugroho W, Kennedy KM. Back to the future: An online OSCE Management Information System for nursing OSCEs. Nurse Educ Today. 2015; 35 (11): 1091-1096.
10. Luimes JD, Labrecque ME. Implementation of electronic objective structured clinical examination evaluation in a nurse practitioner program. J Nurs Educ. 2018; 57 (8): 502-505.

11. Daniels VJ, Strand AC, Lai H, Hillier T. Impact of tablet-scoring and immediate score sheet review on validity and educational impact in an internal medicine residency Objective Structured Clinical Exam (OSCE). Med Teach. 2019; 41 (9): 1039-1044.

12. Snodgrass SJ, Ashby SE, Rivett DA, Russell T. Implementation of an electronic Objective Structured Clinical Exam for assessing practical skills in preprofessional physiotherapy and occupational therapy programs: examiner and course coordinator perspectives. Australas J Educ Technol. 2014; 30 (2): 152-166.

13. Judd T, Ryan A, Flynn E, McColl G. If at first you don't succeed ... adoption of iPad marking for high-stakes assessments. Perspect Med Educ. 2017; 6 (5): 356-361.

14. Monteiro S, Sibbald D, Coetzee K. i-Assess: Evaluating the impact of electronic data capture for OSCE. Perspect Med Educ. 2018; 7 (2): 110-119.

15. Schmitz FM, Zimmermann PG, Gaunt K, Stolze M, Guttormsen Schär S. Electronic rating of objective structured clinical examinations: mobile digital forms beat paper and pencil checklists in a comparative study. In: Holzinger A, Simonic K-M, editors. Information Quality in e-Health. Berlin, Heidelberg: Springer Berlin Heidelberg; 2011. pp. 501-512.

16. Davis FD. Perceived usefulness, perceived ease of use, and user acceptance of information technology. MIS Q. 1989; 13 (3): 319.

17. Granić A, Marangunić N. Technology acceptance model in educational context: A systematic literature review. Br J Educ Technol. 2019; 50 (5): 2572-2593.

18. Currie GP, Sinha S, Thomson F, Cleland J, Denison AR. Tablet computers in assessing performance in a high stakes exam: opinion matters. J R Coll Physicians Edinb. 2017; 47 (2): 164-167.

19. Hochlehnert A, Schultz J-H, Möltner A, Tımbıl S, Brass $\mathrm{K}$, Jünger J. Electronic acquisition of OSCE performance using tablets. GMS Z Med Ausbild. 2015; 32 (4): Doc41.

20. Denison A, Bate E, Thompson J. Tablet versus paper marking in assessment: feedback matters. Perspect Med Educ. 2016; 5 (2): 108-113.

21. Munro AJ, Cumming K, Cleland J, Denison AR, Currie GP. Paper versus electronic feedback in high stakes assessment. J R Coll Physicians Edinb. 2018; 48 (2): 148-152.

22. Harrison CJ, Molyneux AJ, Blackwell S, Wass VJ. How we give personalised audio feedback after summative OSCEs. Med Teach. 2015; 37 (4): 323-326.

23. Ashby SE, Snodgrass SH, Rivett DA, Russell T. Factors shaping e-feedback utilization following electronic objective structured clinical examinations. Nurs Health Sci. 2016; 18 (3): 362-369.

\section{Correspondencia: \\ Carlos Orellano}

Av. Honorio Delgado Núm. 430,

San Martín de Porres. Lima, Perú.

E-mail: carlos.orellano@upch.pe 